\title{
606.
}

\section{ON THE EXPRESSION OF THE COORDINATES OF A POINT OF A QUARTIC CURVE AS FUNCTIONS OF A PARAMETER.}

[From the Proceedings of the London Mathematical Society, vol. vi. (1874-1875), pp. 81-83. Read February 11, 1875.]

THE present short Note is merely the development of a process of Prof. Sylvester's. It will be recollected that the general quartic curve has the deficiency 3 (or it is 4-cursal); the question is therefore that of the determination of the subrational* functions of a parameter which have to be considered in the theory of curves of the deficiency 3.

Taking the origin at a point of the curve, the equation is

$$
(x, y)^{4}+(x, y)^{3}+(x, y)^{2}+(x, y)=0 ;
$$

and writing herein $y=\lambda x$, the equation, after throwing out the factor $x$, becomes

or, say

$$
(1, \lambda)^{4} x^{3}+(1, \lambda)^{3} x^{2}+(1, \lambda)^{2} x+(1, \lambda)=0 ;
$$

where we write for shortness

$$
a x^{3}+3 b x^{2}+3 c x+d=0,
$$

$$
a, b, c, d=(1, \lambda)^{4}, \frac{1}{3}(1, \lambda)^{3}, \frac{1}{3}(1, \lambda)^{2},(1, \lambda)
$$

viz. $a, b, c, d$ stand for functions of $\lambda$ of the degrees $4,3,2$, and 1 respectively.

The equation may be written

$$
(a x+b)^{3}-3\left(b^{2}-a c\right)(a x+b)+a^{2} d-3 a b c+2 b^{3}=0 ;
$$

* The expression "subrational" includes irrational, but it is more extensive; if $Y, X$ are rational functions, the same or different, of $y, x$ respectively and $Y$ is determined as a function of $x$ by an equation of the form $Y=X$, then $y$ is a subrational function of $x$. The notion is due to Prof. Sylvester.

$40-2$ 
viz. writing for a moment $a x+b=2 \sqrt{b^{2}-a c} \cdot u$, this is

Hence, assuming

$$
4 u^{3}-3 u+\frac{a^{2} d-3 a b c+2 b^{3}}{2\left(b^{2}-a c\right) \sqrt{b^{2}-a c}}=0 .
$$

$$
-\cos \phi=\frac{a^{2} d-3 a b c+2 b^{3}}{2\left(b^{2}-a c\right) \sqrt{ } b^{2}-a c},
$$

then we have $4 u^{3}-3 u-\cos \phi=0$; consequently $u$ has the three values $\cos \frac{1}{3} \phi, \cos \frac{1}{3}(\phi+2 \pi)$, $\cos \frac{1}{3}(\phi-2 \pi)$, and we may regard $\cos \frac{1}{3} \phi$ as representing any one of these values.

We have thus $a x+b=2 \sqrt{b^{2}-a c} \cos \frac{1}{3} \phi$, and $y=\lambda x$, giving $x$ and $y$ as functions of $\lambda$ and $\phi$, that is, of $\lambda$. But for their expression in this manner we introduce the irrationality $\sqrt{b^{2}-a c}$, which is of the form $\sqrt{(1, \lambda)^{6}}$, and the trisection or derivation of $\cos \frac{1}{3} \phi$ from a given value of $\cos \phi$; viz. we have, as above, $-\cos \phi$, a function of $\lambda$ of the form

$$
(1, \lambda)^{9} \div(1, \lambda)^{6} \sqrt{(1, \lambda)^{6}}
$$

The equation for $\phi$ may be expressed in the equivalent forms

$$
\begin{aligned}
\sin \phi & =\frac{a \sqrt{-\left(a^{2} d^{2}+4 a c^{3}+4 b^{3} d-6 a b c d-3 b^{2} c^{2}\right)}}{\left(b^{2}-a c\right) \sqrt{b^{2}-a c}} \\
-\tan \phi & =\frac{a \sqrt{-\left(a^{2} d^{2}+4 a c^{3}+4 b^{3} d-6 a b c d-3 b^{2} c^{2}\right)}}{a^{2} d-3 a b c+2 b^{3}}
\end{aligned}
$$

and inasmuch as we have

we may, instead of

$$
2 \sqrt{b^{2}-a c}=-\frac{a^{2} d-3 a b c+2 b^{3}}{\left(b^{2}-a c\right) \cos \phi}
$$

write

$$
a x+b=2 \sqrt{b^{2}-a c} \cos \frac{1}{3} \phi
$$

or, what is the same thing,

$$
a x+b=-\frac{\left(a^{2} d-3 a b c+2 b^{3}\right) \cos \frac{1}{3} \phi}{\left(b^{2}-a c\right) \cos \phi}
$$

$$
=\frac{-\left(a^{2} d-3 a b c+2 b^{3}\right)}{\left(b^{2}-a c\right)\left(4 \cos ^{2} \frac{1}{3} \phi-3\right)} .
$$

The formulæ may be simplified by introducing $\mu$, a function of $\lambda$, determined by the equation

viz. this equation is

$$
c \mu^{2}-2 b \mu+a=0
$$

$$
\frac{1}{3}(1, \lambda)^{2} \mu^{2}-\frac{2}{3}(1, \lambda)^{3} \mu+(1, \lambda)^{4}=0,
$$

so that $(\lambda, \mu)$ may be regarded as coordinates of a point on a nodal quartic curve, or a quartic curve of the next inferior deficiency 2. And we then have

$$
(c \mu-b)=\sqrt{b^{2}-a c}
$$


and consequently

$$
-\cos \phi=\frac{a^{2} d-3 a b c+2 b^{3}}{2(c \mu-b)^{3}}
$$

viz. $\cos \phi$ is given as a rational function of the coordinates $(\lambda, \mu)$; there is, as before, the trisection; and we then have

$$
a x+b=2(c \mu-b) \cos \frac{1}{3} \phi, \quad y=\lambda x,
$$

giving $x$ and $y$ as functions of $\lambda, \mu, \phi$; that is, ultimately, as functions of $\lambda$. I have not succeeded in obtaining in a good geometrical form the relation between the point $(x, y)$ on the given quartic and the point $(\lambda, \mu)$ on the nodal quartic.

Reverting to the expression of $\tan \phi$, it may be remarked that $a=0$ gives the values of $\lambda$ which correspond to the four points at infinity on the given quartic curve; $a^{2} d^{2}+4 a c^{3}+4 b^{3} d-6 a b c d-3 b^{2} c^{2}=0$, the values corresponding to the ten tangents from the origin; and $a^{2} d-3 a b c+2 b^{3}=0$, the values corresponding to the nine lines through the origin, which are each such that the origin is the centre of gravity of the other three points on the line.

I take the opportunity of mentioning a mechanical construction of the Cartesian. The equation $r^{\prime}=-A \cos \theta-N$ represents a limaçon (which is derivable mechanically from the circle $r^{\prime}=-A \cos \theta$ ), and if we effect the transformation $r^{\prime}=r+\frac{B}{r}$, the new curve is $r+\frac{B}{r}+A \cos \theta+N=0$; that is, $r^{2}+r(A \cos \theta+N)+B=0$, which is, in fact, the equation of a Cartesian. The assumed transformation $r^{\prime}=r+\frac{B}{r}$ can be effected immediately by a Peaucellier cell. 